OPEN ACCESS

Edited by:

Christoph Aebi,

University Children's Hospital

Bern, Switzerland

Reviewed by:

Nicole Ritz,

University Children's Hospital

Basel, Switzerland

Rinawati Rohsiswatmo,

RSUPN Dr. Cipto Mangunkusumo,

Indonesia

*Correspondence:

Novel N. Chegou

novel@sun.ac.za

†These authors have contributed equally to this work

Specialty section:

This article was submitted to Pediatric Infectious Diseases,

a section of the journal

Frontiers in Pediatrics

Received: 16 April 2019 Accepted: 02 September 2019

Published: 25 September 2019

Citation:

Manyelo CM, Solomons RS,

Snyders Cl, Mutavhatsindi $\mathrm{H}$,

Manngo PM, Stanley K, Walzl G and Chegou NN (2019) Potential of Host

Serum Protein Biomarkers in the

Diagnosis of Tuberculous Meningitis in

Children. Front. Pediatr. 7:376.

doi: 10.3389/fped.2019.00376

\section{Potential of Host Serum Protein Biomarkers in the Diagnosis of Tuberculous Meningitis in Children}

\author{
Charles M. Manyelo ${ }^{1 \dagger}$, Regan S. Solomons ${ }^{2 \dagger}$, Candice I. Snyders ${ }^{1}$, Hygon Mutavhatsindi ${ }^{1}$, \\ Portia M. Manngo ${ }^{1}$, Kim Stanley ${ }^{1}$, Gerhard Walzl ${ }^{1}$ and Novel N. Chegou ${ }^{1 *}$ \\ ${ }^{1}$ DST-NRF Centre of Excellence for Biomedical Tuberculosis Research, South African Medical Research Council Centre for \\ Tuberculosis Research, Division of Molecular Biology and Human Genetics, Department of Biomedical Sciences, Faculty of \\ Medicine and Health Sciences, Stellenbosch University, Cape Town, South Africa, ${ }^{2}$ Department of Paediatrics and Child \\ Health, Faculty of Medicine and Health Sciences, Stellenbosch University, Cape Town, South Africa
}

Background: Tuberculous meningitis (TBM) is the most severe form of tuberculosis and results in high morbidity and mortality in children. Diagnostic delay contributes to the poor outcome. There is an urgent need for new tools for the rapid diagnosis of TBM, especially in children.

Methods: We collected serum samples from children in whom TBM was suspected at a tertiary hospital in Cape Town, South Africa. Children were subsequently classified as having TBM or no TBM using a published uniform research case-definition. Using a multiplex cytokine array platform, we investigated the concentrations of serum biomarkers comprising biomarkers that were previously found to be of value in the diagnosis of adult pulmonary TB (CRP, SAA, CFH, IFN- $\gamma$, IP-10, Apo-Al, and transthyretin) plus other potentially useful host biomarkers as diagnostic candidates for TBM.

Findings: Out of 47 children included in the study, 23 (48.9\%) had a final diagnosis of TBM and six were HIV infected. A modified version of the adult 7-marker biosignature in which transthyretin was replaced by NCAM1, diagnosed TBM in children with AUC of 0.80 (95\% Cl, 0.67-0.92), sensitivity of 73.9\% (95\% Cl, 51.6-89.8\%) and specificity of $66.7 \%(95 \% \mathrm{Cl}, 44.7-84.4 \%)$, with the other six proteins in the signature (CRP, IFN- $\gamma$, IP-10, CFH, Apo-A1, and SAA) only achieving and AUC of 0.75 (95\% Cl, 0.61-0.90) when used in combination. A new childhood TBM specific 3-marker biosignature (adipsin, $A \beta 42$, and IL-10) showed potential in the diagnosis of TBM, with AUC of $0.84(95 \%$ $\mathrm{Cl}, 0.73-0.96)$, sensitivity of $82.6 \%$ (95 Cl, 61.2-95.0\%) and specificity of $75.0 \%$ (95\% $\mathrm{Cl}, 53.3-90.2 \%)$ after leave-one-out cross validation.

Conclusion: A previously described adult 7-marker serum protein biosignature showed potential in the diagnosis of TBM in children. However, a smaller childhood TBM-specific 3-marker signature demonstrated improved performance characteristics. Our data indicates that blood-based biomarkers may be useful in the diagnosis of childhood TBM and requires further validation in larger cohort studies.

Keywords: tuberculosis, meningitis, biomarker, sensitivity and specificity, diagnosis, early diagnosis, immunologic tests, child 


\section{INTRODUCTION}

Globally, tuberculosis (TB) is currently one of the top 9 causes of death, ranking above HIV and malaria (1). According to the World Health Organisation (WHO) $2018 \mathrm{~TB}$ report, 10\% of all reported TB cases in 2017 were in children (2), and 230 000 children died from TB (1). About $20-25 \%$ of pediatric TB cases are extra pulmonary TB (EPTB), including tuberculous meningitis (TBM) (3). In a clinical and laboratory surveillance study over a 1 year period in the Western Cape Province of South Africa almost one-fifth of children with $\mathrm{TB}$ had a disseminated form (TB meningitis or miliary TB) (4).

TBM is a central nervous system infection caused by Mycobacterium tuberculosis (M. $t b)$ and it is the most severe form of tuberculosis, with high morbidity and mortality $(5,6)$. The rate of TBM is higher among children than adults (7). A study in South Africa reported that TBM is the most common form of bacterial meningitis among children (8). Outcomes of TBM in children include death in up to $50 \%$ of cases and neurological sequelae in up to $53.9 \%$ of survivors $(9,10)$. The poor outcomes of TBM are mainly due to delayed diagnosis and late initiation of anti-tuberculosis therapy (11). The improvement of case detection and early administration of curative treatment are key in the management of pediatric tuberculosis (12). However, the diagnosis of TBM in children is challenging, due to sub-optimal performance of the currently available laboratory diagnostic methods. Smear microscopy remains the most widely used test for bacteriological confirmation of TB in clinical specimens, particularly in resource-constrained settings (13). Smear microscopy has poor sensitivity of about $10-20 \%$ in diagnosing TBM (9). Although $M$. $t b$ culture, the gold standard test for diagnosing TB disease has a relatively high sensitivity $(60-70 \%)$ for diagnosing TBM in comparison to smear microscopy, its turnaround time is up to 42 days (9). Furthermore, culture is expensive, prone to contamination and requires extensive laboratory infrastructure, which is not often available in resource-constrained settings (14). The GeneXpert MTB/RIF test ${ }^{\circledR}$, arguably the most important recent advance in TB diagnosis, yields results within $2 \mathrm{~h}$ and detects resistance to rifampicin as a proxy for the presence of MDR strains. However, GeneXpert has several well-publicized shortcomings including low negative predictive value, cost effectiveness and the requirement for technical infrastructure $(13,15)$.

The GeneXpert cannot exclude TBM due to its imperfect sensitivity and negative predictive value (16). A study conducted in Uganda reported sensitivity of $28 \%$ when using $2 \mathrm{~mL}$ of uncentrifuged CSF, and the sensitivity improved to $72 \%$ when a large volume (6 mL) of centrifuged CSF was used (17), suggesting that high volumes of CSF are required to obtain a positive TBM diagnosis using Xpert. The GeneXpert Ultra has been shown to overcome some of the shortcomings of GeneXpert MTB/RIF as it showed improved sensitivity of $95 \%$ and negative predictive value of $99 \%$ in a more recent study on HIV positive adults with TBM (16). However, there are concerns over lowered specificity and positive predictive value compared to GeneXpert MTB/RIF, which require prospective testing in a large population including children and those that are HIV-uninfected $(16,18,19)$. The main common limitation to the above-described tests is the difficulty in obtaining the diagnostic sample (CSF).

The diagnosis of TBM is mostly based on a combination of suggestive clinical findings, multiple suggestive laboratory tests on the CSF, supportive features on brain imaging findings and the exclusion of other possible causes of meningitis. These criteria are unreliable as individual tests, and often require referral of children to tertiary healthcare facilities for the performance of advanced testing, due to the unavailability of most of the relevant techniques in primary and secondary healthcare facilities, leading to further delays in the initiation of treatment (20). Consequently, children seen at primary and secondary healthcare facilities often have multiple missed opportunities; up to six visits before eventual diagnosis of TBM is made (20). There is an urgent need for new TBM diagnostic tools suitable for use in children at the point of care or bedside.

Host biomarker-based tests may be valuable in the diagnosis of TBM as they have previously been shown to be potentially useful in other extrapulmonary forms of TB (21), and may be easily converted into point-of-care or bedside tests $(22,23)$. Most studies investigating host biomarkers for diagnosis of neurological disorders have focused on CSF as a biological specimen from the site of infection (24), including in the diagnosis of TBM $(25,26)$. Despite the potential of CSF-based host biomarkers in the diagnosis of TBM, the collection of CSF requires lumbar puncture, an invasive procedure which requires special skill and training and might not be readily available, leading to delay in diagnosis.

As several studies have shown the potential of blood-based host protein biomarkers in the diagnosis of TB disease, albeit mostly adult pulmonary TB-based studies thus far $(27,28)$, we hypothesized that blood-based host protein biosignatures may be useful in the diagnosis of TBM in children. The aim of the present study was to ascertain whether host biomarkers that have shown potential in the diagnosis of adult pulmonary TB in serum and plasma samples $(27,28)$ possessed diagnostic potential for childhood TBM. We were specifically interested in evaluating the performance of a previously established adult seven-marker serum protein biosignature (CRP, transthyretin, IFN- $\gamma$, CFH, Apo-AI, IP-10, and SAA) (28) as a tool for the diagnosis of childhood TBM, and to also evaluate the potential of other host biomarkers.

\section{MATERIALS AND METHODS}

\section{Study Participants}

Participants enrolled into the present study were children who presented with signs and symptoms suggestive of meningitis and requiring CSF examination for routine diagnostic purposes at the Tygerberg Academic Hospital in Cape Town, South Africa between November 2016 and November 2017 as previously described (29). Children were eligible for participation in the study if they were between the ages of 3 months and 13 years, provided that written informed consent was obtained from the parents or legal guardians. Assent was obtained from children older than 7 years if they had a normal level of consciousness i.e., a Glasgow Coma Score (GCS) of 15/15. The study was approved 
by the Health Research Ethics Committee of the University of Stellenbosch, Tygerberg Academic Hospital, and the Western Cape Provincial Government.

After collection of specimens for routine diagnostic purposes, an additional $1 \mathrm{ml}$ of blood was collected into a BD Vacutainer ${ }^{\circledR}$ serum tube and processed within an average of $2 \mathrm{~h}$ from collection, for the purposes of the present study. Blood samples were centrifuged at $1,200 \times \mathrm{g}$ for $10 \mathrm{~min}$, followed by aliquoting of serum and storage at $-80^{\circ} \mathrm{C}$ until analyzed.

\section{Diagnostic Assessment}

As previously described (29), a comprehensive clinical examination was performed on all patients by a specialist pediatric neurologist. Following routine clinical investigations, computed tomography (CT) of the brain, air-encephalography, and magnetic resonance (MR) imaging were performed as clinically indicated. CSF was obtained through lumbar puncture and investigations including appearance and color determination, differential cell counts, CSF protein, and glucose levels were done. After CSF centrifugation, the following procedures were done: Gram staining, India ink examination, culture of the centrifuged sediment on blood agar plates (for pyogenic bacteria), Auramine "O" staining and fluorescence microscopy, culture using the mycobacterium growth indicator tubes (MGIT) ${ }^{\mathrm{TM}}$ (Becton and Dickinson) and examination for $M$. $t b$ DNA using the HAIN Genotype MTBDRplus kit (Hain Life science $\mathrm{GmbH}$, Germany). Other routinely investigated parameters including serum glucose level and viral PCR were done. All data generated from the study were recorded in a study specific REDCap web-based database.

\section{Classification of Study Participants}

Patients were classified as probable or definite TBM according to a uniform research case definition (30) based on a scoring system consisting of clinical criteria, CSF criteria, cerebral imaging criteria, evidence of extraneural TB and exclusion of alternative diagnoses. TBM was classified as "probable" when patients scored $\geq 12$ when neuroimaging was available and $\geq 10$ when neuroimaging was unavailable. Following the definition by Marais et al. (30), a diagnosis of definite TBM was made if there was detection of the M. $t b$ bacilli in the CSF by one of the following tests: acid-fast bacilli, culture, or nucleic acid amplification tests (NAATs). For the purposes of this study, the TBM group included probable TBM and definite TBM, whereas the no-TBM group included children with other types of meningitis including bacterial and viral meningitis, and a wide range of other diagnoses as mentioned in Table $\mathbf{1}$ and also previously reported (29).

\section{Immunoassays}

We evaluated the concentrations of biomarkers assessed in our previous study (29) in serum samples obtained from the same study participants. As reported in our previous study, the concentrations of 69 host biomarkers including six of the proteins comprising the previously established adult seven-marker serum protein biosignature (CRP, SAA, complement factor $\mathrm{H}, \mathrm{IFN}-\gamma$, IP-10, and Apo AI) (28), were evaluated by ELISA (cathelicidin LL-37) or using the Luminex platform (all other biomarkers). Transthyretin, one of the biomarkers comprising the previously established adult sevenmarker signature was not assessed due to discontinued supply from the manufacturer.

Serum cathelicidin LL-37 levels were evaluated using an ELISA kit purchased from Elabscience Biotechnology Inc. (Catalog \#E-EL-H2438). Experiments were done according to the procedure recommended by the manufacturer after which optical densities (OD) were read at $450 \mathrm{~nm}$ by an automated microplate reader (iMark ${ }^{\mathrm{TM}}$ Microplate Reader, Bio Rad Laboratories). The mean OD of the blank wells was subtracted from the OD of the sample wells and the background-corrected ODs used for statistical analysis.

Serum levels of CCL1(I-309), CCL2(MCP-1), CCL3(MIP$1 \alpha)$, CCL4(MIP-1 $\beta$ ), CD40 ligand (CD40L), CXCL8(IL-8), CXCL9(MIG), CXCL10(IP-10), granulocyte colony-stimulating factor (G-CSF), granulocyte-macrophage colony-stimulating factor(GM-CSF), interferon (IFN)- $\gamma$, interleukin (IL)-1 $\beta$, IL-10, IL-12/23(p40), IL-17A, IL-13, IL-21, IL-4, IL-6, IL-7, matrix metalloproteinase (MMP)-1, MMP-8, transforming growth factor (TGF)- $\alpha$, tumor necrosis factor (TNF)- $\alpha$, soluble neural cell adhesion molecule (sNCAM-1/CD56), MMP-7, VEGF-A, ferritin and MMP-9 were assessed in Luminex kits purchased from R\&D Systems Inc. (Bio-Techne), Minneapolis, USA wherease those of apolipoprotein (Apo)-AI, Apo-CIII, complement C3, complement factor $\mathrm{H}$, BDNF, cathepsin $\mathrm{D}$, soluble intracellular adhesion molecule (sICAM)1, myeloperoxidase (MPO), platelet derived growth factor (PDGF)-AA, CCL5(RANTES), PDGF-AB/BB, soluble vascular adhesion molecule (sVCAM-1), plasminogen activator inhibitor (PAI)-1(total), S100 calcium-binding protein B (S100B), amyloid beta 1-40 (Ab40), Ab42, soluble receptor for advanced glycation end products (sRAGE), Glial cell-derived neurotrophic factor (GDNF), C reactive protein (CRP), alpha-2-antitrypsin (A1AT), pigment epithelium-derived factor (PEDF), serum amyloid $\mathrm{P}$ (SAP), CCL18(MIP-4/PARC), complement C4 (CC4), CC2, CC4b, CC5, CC5a, CC9, complement factor D (adipsin/CFD), mannose binding lectin (MBL), complement factor 1 (CF1), sPselectin, von Willebrand factor-cleaving protease (ADAMTS13), D-DIMER, growth differentiation factor (GDF)-15, myoglobin, lipocalin2 (NGAL), and serum amyloid A (SAA) were evaluated in kits purchased form Merck Millipore, Billerica, MA, USA.

All biomarkers were evaluated following the instructions of the respective kit manufacturers (R\&D Systems and Merck Millipore, respectively) in a blinded manner. All experiments were performed on the Bio Plex platform (Bio Rad Laboratories, Hercules, USA) in an ISO15189 accredited laboratory. Data acquisition and analysis of median fluorescent intensity was done using the Bio Plex Manager Version 6.1 software (Bio Rad Laboratories). The values of analytes in the quality control reagents evaluated with the samples were within their expected ranges.

\section{Statistical Analysis}

Data were analyzed using Statistica (TIBCO Software Inc., CA, USA), and GraphPad Prism version 6 (Graphpad 
TABLE 1 | Clinical and demographic characteristics of children included in the study.

\begin{tabular}{|c|c|c|c|c|c|}
\hline & $\begin{array}{l}\text { All, No. } \\
\text { (\%) }\end{array}$ & $\begin{array}{c}\text { Definite TBM, No. } \\
(\%)\end{array}$ & $\begin{array}{c}\text { Non-confirmed TBM, } \\
\text { No. (\%) }\end{array}$ & $\begin{array}{l}\text { All TBM, } \\
\text { No. (\%) }\end{array}$ & $\begin{array}{l}\text { No-TBM\#, } \\
\text { No. (\%) }\end{array}$ \\
\hline Number of participants & 47 & $3(6.4)$ & $20(42.6)$ & $23(48.9)$ & $24(51.1)$ \\
\hline Median age in months (IQR) & $22.0(10.5-57.0)$ & $58.0(36.5-101.5)$ & $17.5(10.3-31.5)$ & $18.0(11.0-40.0)$ & $30.0(9.0-96.0)$ \\
\hline Males, $n(\%)$ & $30(63.8)$ & 1 (33.3) & $12(60.0)$ & $13(56.5)$ & $17(70.8)$ \\
\hline HIV Positive, $n /$ no tested & $6 / 37$ & $0 / 2$ & $0 / 19$ & $0 / 21$ & $6 / 15$ \\
\hline BCG done, $n(\%)$ & $33(70.2)$ & $0(0.0)$ & $16(80.0)$ & $17(73.9)$ & $16(66.7)$ \\
\hline TB contact in history & $14(29.8)$ & $0(0.0)$ & $7(35.0)$ & 7 (30.4) & $7(29.2)$ \\
\hline \multicolumn{6}{|l|}{ Presenting symptoms } \\
\hline Fever & $17(36.2)$ & $2(66.7)$ & $7(35.0)$ & $10(43.5)$ & $7(29.2)$ \\
\hline Vomiting & $12(25.5)$ & $0(0.0)$ & $6(30.0)$ & $7(30.4)$ & $5(20.8)$ \\
\hline Weight loss & $10(21.3)$ & 1 (33.3) & $6(30.0)$ & 7 (30.4) & $3(12.5)$ \\
\hline Seizures & $18(38.3)$ & 1 (33.3) & $9(45.0)$ & 9 (39.1) & 9 (37.5) \\
\hline Cough & $16(34.0)$ & 1 (33.3) & $6(30.0)$ & 9 (39.1) & $7(29.2)$ \\
\hline Altered consciousness & $13(27.7)$ & 1 (33.3) & $6(30.0)$ & $6(26.1)$ & 7 (29.2) \\
\hline Symptom duration, days & 15.0 & 18.5 & 25.8 & 9.4 & 5.6 \\
\hline \multicolumn{6}{|l|}{ Clinical signs } \\
\hline Raised intracranial pressure & $10(21.3)$ & 1 (33.3) & $5(25.0)$ & $5(21.7)$ & $5(20.8)$ \\
\hline Hemiplegia & $13(27.7)$ & 1 (33.3) & $9(45.0)$ & 7 (30.4) & $6(25.0)$ \\
\hline
\end{tabular}

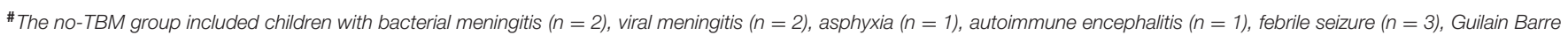

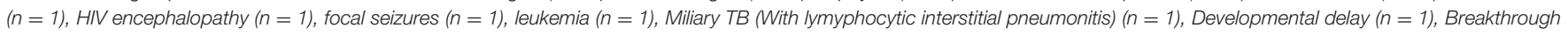



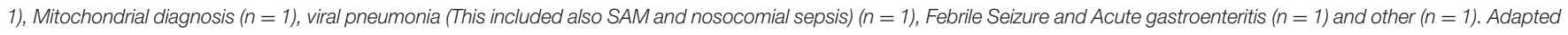

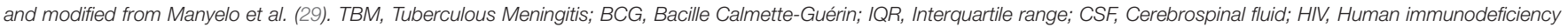
virus. Unless otherwise stated, $n=47$.

software, CA, USA). The Mann Whitney U test was used to compare the differences in the concentrations of host biomarkers between the TBM and the no-TBM groups. The receiver operator characteristics (ROC) curve analysis procedure was used to assess the diagnostic accuracy of individual host biomarkers for TBM. Optimal cut-off values and associated sensitivities and specificities were selected based on the Youden's index (31). The utility of combinations of biomarkers in the diagnosis of TBM was ascertained by general discriminant analysis (GDA), followed by leave-oneout cross validation. Data were log-transformed to prior to GDA.

\section{RESULTS}

A total of 47 children who presented with signs and symptoms strongly suggestive of TBM were included in the study, 30 $(63.8 \%)$ of whom were males (Figure 1). The mean age of all the children was 22 months (interquartile range: 10.5-57) and six out of 37 with known HIV status (16.2\%) were HIV infected. Bacillus Calmette-Guérin (BCG) vaccination is routinely administered in the high TB burdened setting where study participants were recruited. Out of the 47 study participants, 33 (70.2\%) had documented evidence of BCG vaccination. The performance of the biomarkers was assessed regardless of BCG vaccination status. Using a composite reference standard based on a uniform research case definition of TBM (30), 23 of the children were diagnosed with definite $(n=3)$ or probable
( $n=20)$ TBM. The 24 children without TBM included children with bacterial meningitis $(n=2)$, viral meningitis $(n=2)$, and children with other diagnoses as described in Tables 1, 2.

\section{Application of the Previously Established Adult 7-Marker Serum Protein Biosignature in the Diagnosis of TBM in Children}

We aimed to assess the performance of the previously described adult pulmonary TB seven-marker (CRP, IFN- $\gamma$, IP-10, CFH, Apo-A1, SAA and transthyretin) in the diagnosis of TBM in children. However, one of the key biomarkers comprising the 7marker serum biosignature, transthyretin, was not available due to discontinued supply from the reagent manufacture (Merck). When the concentrations of the six available markers (CRP, IFN$\gamma$, IP-10, CFH, Apo-A1, and SAA), from the adult pulmonary TB seven-marker signature (28) were evaluated in serum samples from children with TBM vs. those without TBM individually, significant differences were obtained for CFH only. After ROC curve analysis, the most useful individual marker from this signature, as determined by AUC was CFH (Table 3). When used in combination, the AUC for this 6-marker model in the diagnosis of TBM was 0.75 (95\% CI, 0.61-0.90); corresponding to sensitivity of $69.6 \%$ (95\% CI, 47.1-86.8\%) and specificity of $62.5 \%$ (95\% CI, 40.6-81.2\%). After leave-one-out cross validation, the 6-marker combination diagnosed TBM with sensitivity of $65.2 \%$ (95\% CI, 42.7-83.6\%) and specificity of 54.2\% (95\% CI, 32.8$74.5 \%)$. When transthyretin was replaced by NCAM1, another 
TABLE 2 | Overview of scoring criteria in children with "definite," "probable," and "no" TBM.

\begin{tabular}{|c|c|c|c|}
\hline & Definite TBM n/N (\%) & Probable TBM n/N (\%) & No TBM n/N (\%) \\
\hline Total number & 3 & 20 & 24 \\
\hline Symptom duration $>5$ days & 1/3 (33) & $13 / 20(65)$ & $7 / 24(29)$ \\
\hline $\begin{array}{l}\geq 1 \text { week of: weight loss/poor weight gain, night sweats or persistent } \\
\text { cough }>2 \text { weeks }\end{array}$ & 1/3 (33) & $9 / 20(45)$ & 3/24 (13) \\
\hline History of recent TB contact or positive TST or IGRA & $0 / 3(0)$ & 9/20 (45) & $5 / 24(21)$ \\
\hline Altered consciousness & $1 / 3(33)$ & $8 / 20(40)$ & 4/24 (17) \\
\hline CSF clear & $3 / 3(100)$ & 20/20 (100) & 20/24 (83) \\
\hline CSF cells: $10-500$ per $\mu \mathrm{l}$ & $3 / 3(100)$ & $15 / 20(75)$ & $1 / 24(4)$ \\
\hline CSF lymphocyte predominance (>50\%) & $3 / 3(100)$ & $14 / 20(70)$ & 8/24 (33) \\
\hline CSF protein concentration $>1 \mathrm{~g} / \mathrm{L}$ & $3 / 3(100)$ & 13/20 (65) & 3/24 (13) \\
\hline Tuberculoma (CT and/or MRI) & $0 / 3(0)$ & 4/20 (20) & 0/24 (0) \\
\hline Infarct (CT and/or MRI) & $2 / 3(67)$ & $8 / 20(40)$ & 3/24 (13) \\
\hline Pre-contrast basal hyperdensity (CT) & $1 / 3(33)$ & 6/20 (30) & $0 / 24(0)$ \\
\hline CXR suggestive of active TB & 2/3 (67) & $8 / 20(40)$ & 7/24 (29) \\
\hline CXR suggestive miliary TB & 0/3 (0) & $5 / 20(25)$ & 2/24 (8) \\
\hline Extraneural radiological TB & $0 / 3(0)$ & $0 / 20(0)$ & $0 / 24(0)$ \\
\hline Extraneural $M$. tuberculosis confirmation & $1 / 3(33)$ & $6 / 20(30)$ & 4/24 (17) \\
\hline
\end{tabular}

TBM, tuberculous meningitis; BM= bacterial meningitis; TB, tuberculosis; TST, tuberculin skin test; IGRA, interferon gamma-release assay; CSF, cerebrospinal fluid; gluc, glucose; CT, computed tomography; MRI, magnetic resonance imaging; CXR, chest radiograph. Unless otherwise stated, $n=47$.

candidate biomarker that showed potential in a previous adult study (27), the modified 7-marker biosignature (CRP, IFN- $\gamma$, IP-10, CFH, Apo-A1, SAA, and NCAM1) diagnosed TBM with improved AUC of 0.80 (95\% CI, 0.67-0.92); corresponding to sensitivity of $73.9 \%$ (95\% CI, 51.6-89.8\%) and specificity of 66.7\% (95\% CI, 44.7-84.4\%) (Figure 2). After leave-one-out cross validation, the modified 7-marker biosignature diagnosed TBM with sensitivity of $60.9 \%$ (95\% CI, 38.5-80.3\%) and specificity of $58.3 \%$ (95\% CI, 36.6-77.9\%), with positive and negative predictive values of $58.3 \%$ (95\% CI, 44.1-71.4) and 60.9\% (95\% CI, 45.8-74.1), respectively.

\section{Utility of All Individual Host Serum Protein Biomarkers in the Diagnosis of TBM}

When the concentrations of all individual host biomarkers (total of 69) evaluated in this study were compared between children with and those without TBM, regardless of whether they were part of the adult 7-marker signature or not, using the Mann Whitney $U$ test, the median levels of 16 including sVCAM-1, CCL2, IL-4, TNF- $\alpha$, CCL4, adipsin, SAP, CC5, GCSF, IL-10, Apo-CIII, IL-17A, PAI-1, PDGF AB/BB, MBL, and NCAM-1 were significantly different $(p<0.05)$ between the two groups, with differences in the concentrations of five markers (CC4b, MMP-1, CXCL8, CC4, sRAGE) showing trends for differences between the two groups $(0.05<p$ $\leq$ 0.09). The concentrations of SAP, CC5, Apo-CIII, PAI-1,
PDGF-AB/BB, and MBL were significantly higher in samples from children with TBM whereas those of sVCAM-1, CCL2, IL-4, TNF- $\alpha$, CCL4, adipsin, G-CSF, IL-10, IL-17A, and NCAM-1 were higher in samples from children without TBM (Supplementary Table 1). When the diagnostic potentials of individual serum biomarkers were assessed by ROC curve analysis, 13 of these markers showed promise as ascertained by AUC $\geq 0.70$ (Supplementary Table 1, Figure 3). When only HIV negative children were considered, there were improvements in the performances of other host markers including MMP-1 and IL-7 whereas median levels of six including IL-10, MBL, sRAGE, CC4, CC4b, and NCAM-1 were no longer significantly different (Data not shown).

\section{Identification of a New 3-Marker Signature for the Diagnosis of TBM}

When the data obtained from all study participants were fitted into the General Discriminant Analysis (GDA) models regardless of HIV status and regardless of whether biomarkers were part of the adult 7-marker signature or not, optimal prediction of TBM was shown to be achieved with a combination of three markers. The most accurate three-marker biosignature comprising of adipsin, A $\beta 42$ and IL-10 diagnosed TBM with AUC of 0.84 (95\% CI, 0.73-0.96); corresponding to a sensitivity of $82.6 \%$ (95\% CI, 61.2-95.0\%) and specificity of $75.0 \%$ (95\% CI, 53.390.2\%) (Figure 4). After leave-one-out cross validation, there 


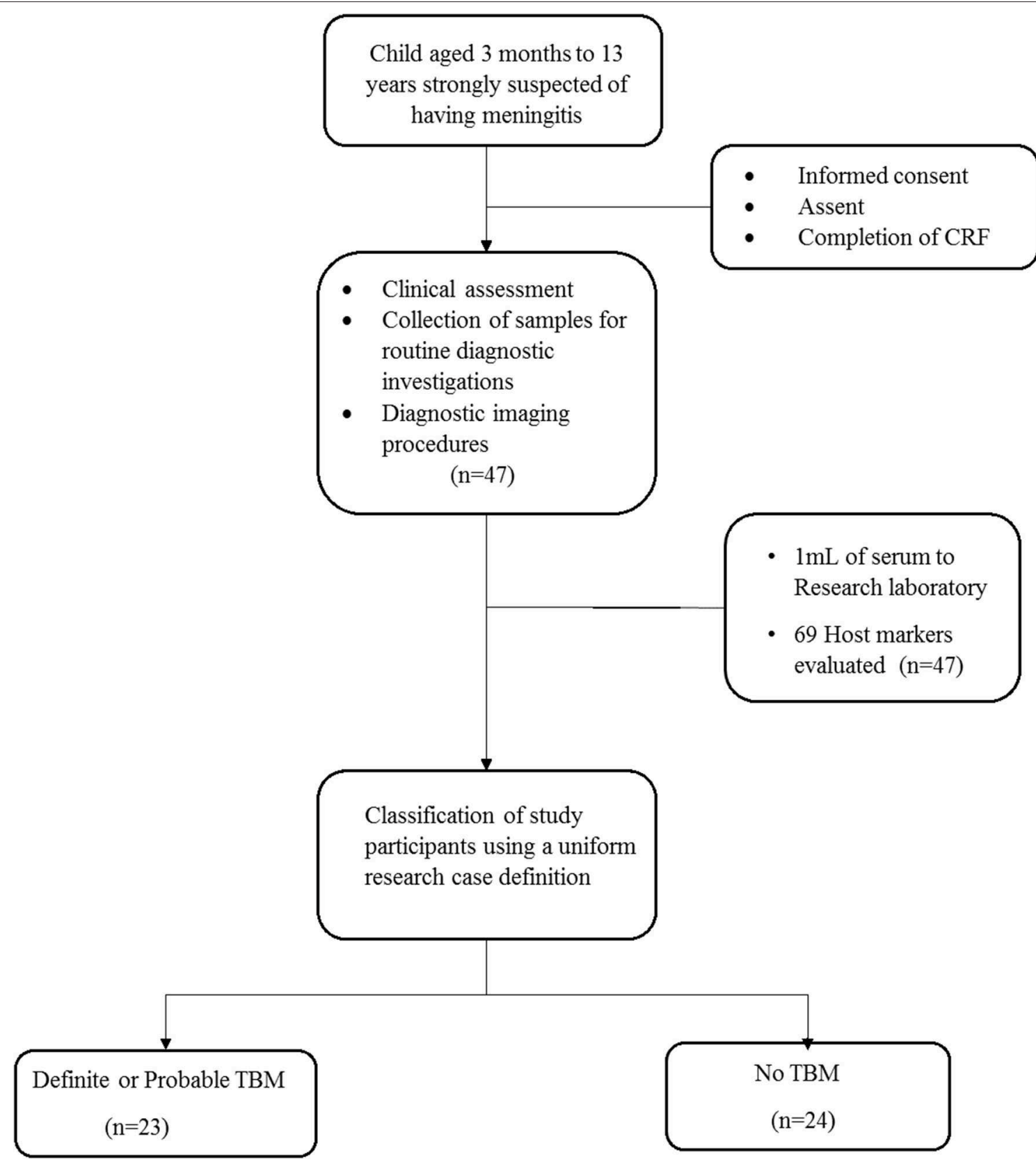

FIGURE 1 | Flow chart showing the study design and classification of study participants. CRF, case report form; TBM, Tuberculous meningitis; No-TBM, Individuals presenting with symptoms and investigated for TB but TBM ruled out. The No TBM group included bacterial meningitis $(n=2)$, viral meningitis $(n=2)$ and children with other diagnoses (Table 1). Adapted from Manyelo et al. (29).

was no change in the sensitivity of the 3-marker biosignature, whereas the specificity dropped to $70.8 \%$ (95 CI, 48.9-87.4\%). The positive and negative predictive values of the biosignature were $73.1 \%$ (95\% CI, 58.6-83.9) and $81.0 \%$ (95\% CI, 62.791.5), respectively after leave-one-out cross validation. Further optimization of the biosignature through the selection of better cut-off values yielded both improved sensitivity and specificity of $83 \%$.

\section{Differential Expression of Host Biomarkers in Serum, Compared to CSF}

Considering that all biomarkers evaluated in the current study were previously evaluated in CSF samples from the same children (29), we compared expression of these proteins between CSF and serum. We observed that the levels of 18 proteins including $A \beta 40, A \beta 42$, MIP- $1 \alpha$, IL-8, IFN- $\gamma$, IL-10, 
TABLE 3 | Usefulness of analytes comprising the previously established adult 7-marker serum protein biosignature in the diagnosis of TBM in children.

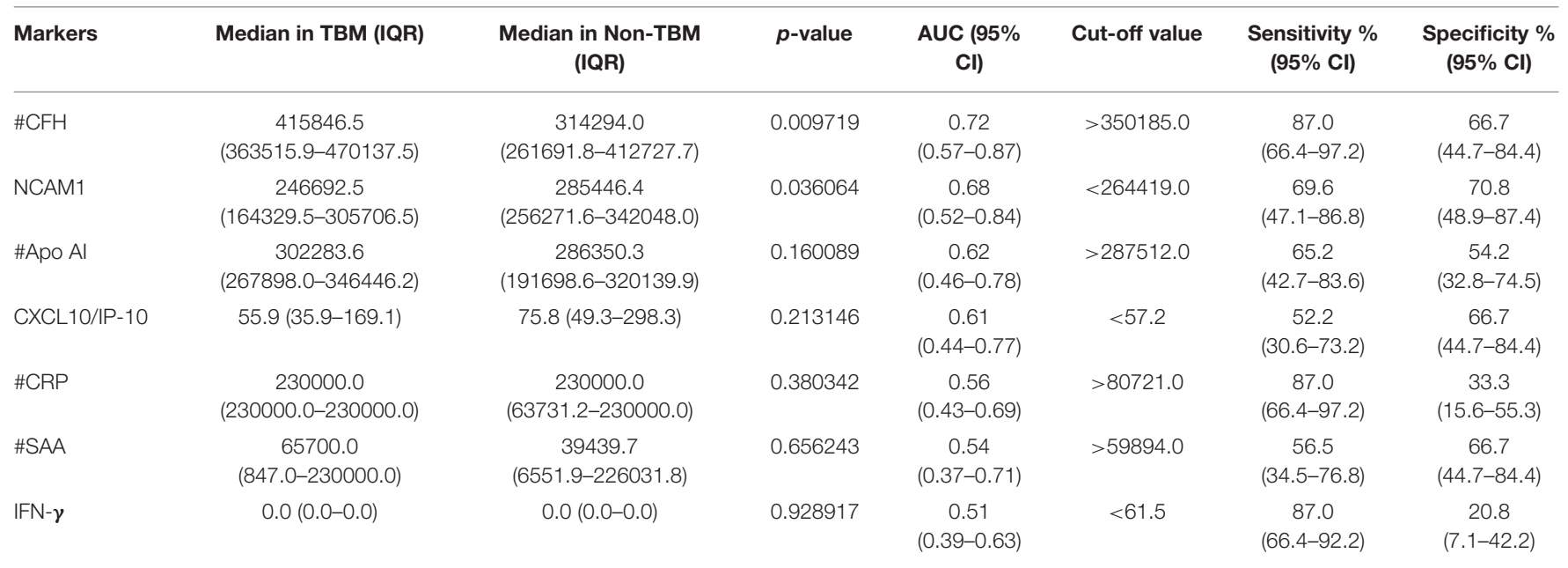

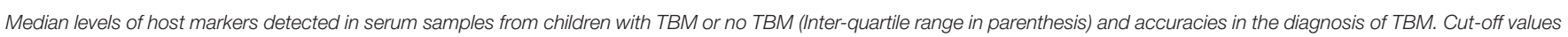
and associated sensitivities and specificities were selected based on the Youden's index. \#values shown are in ng/ml, values for all other host markers are in pg/ml.

\section{A}

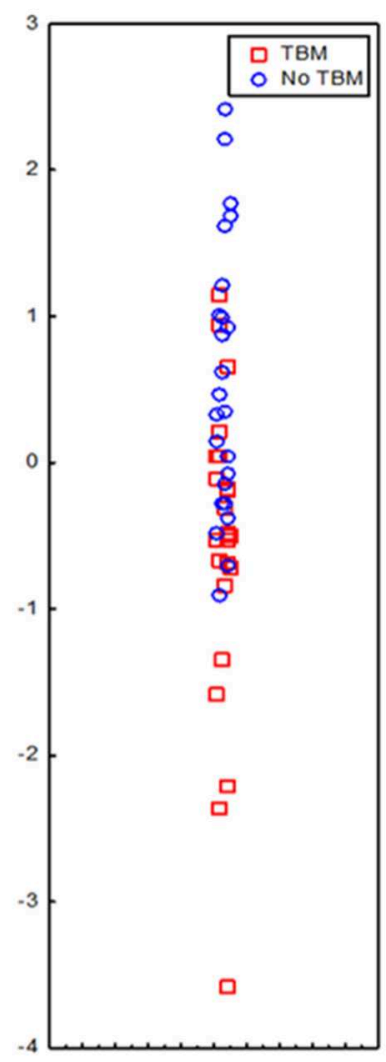

B

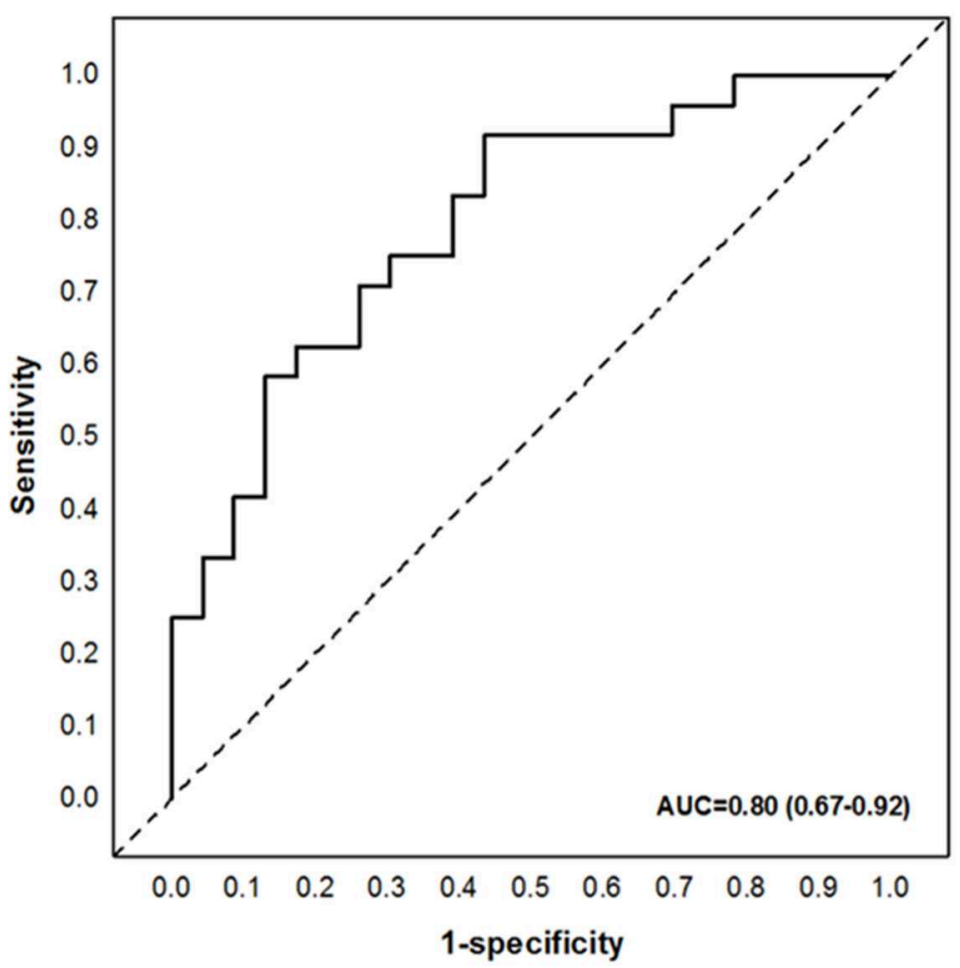

FIGURE 2 | Accuracy of the modified 7-marker serum protein biosignature (CRP, IFN- $\gamma$, IP-10, CFH, Apo-A1, SAA, and NCAM1) in the diagnosis of TBM. Scatter plot showing the ability of the 7-marker signature to classify children as TBM or no TBM (A). ROC curve showing the accuracy of the 7-marker biosignature (B). Red squares; children with TBM; blue circles: children with No TBM. 


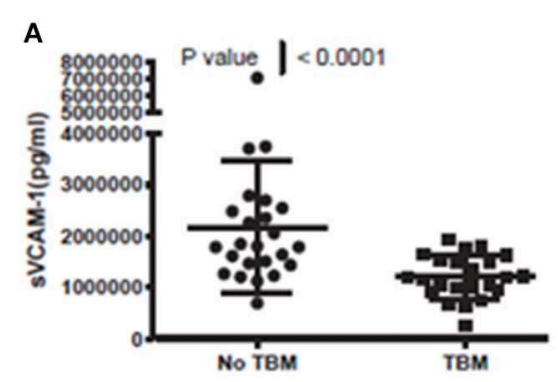

D

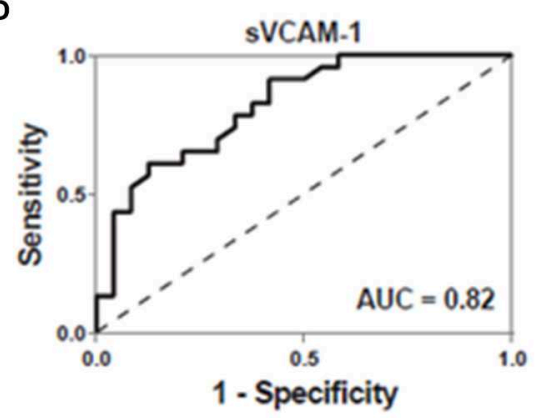

G

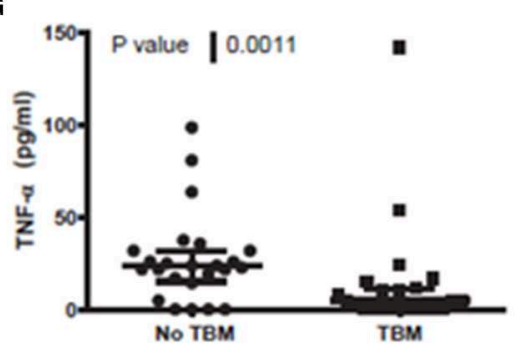

J





E

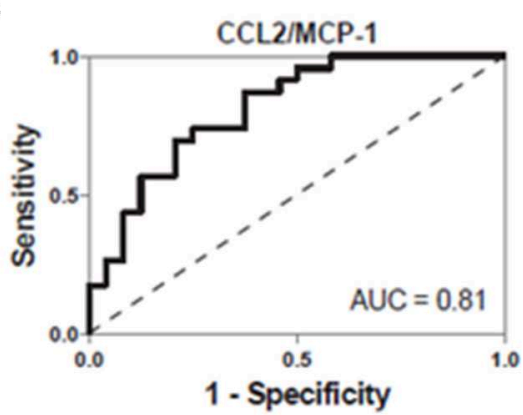

H

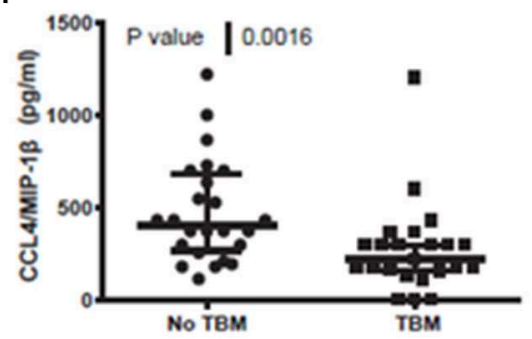

K

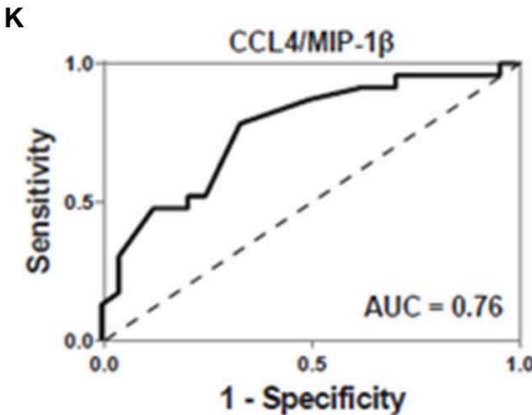

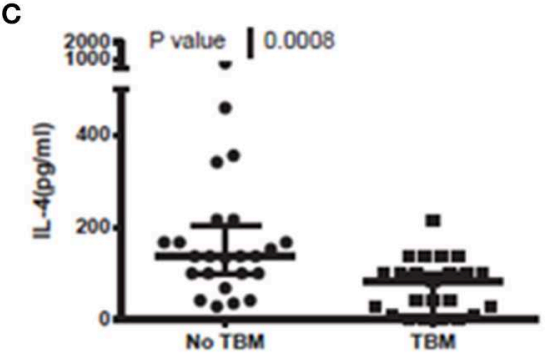

F
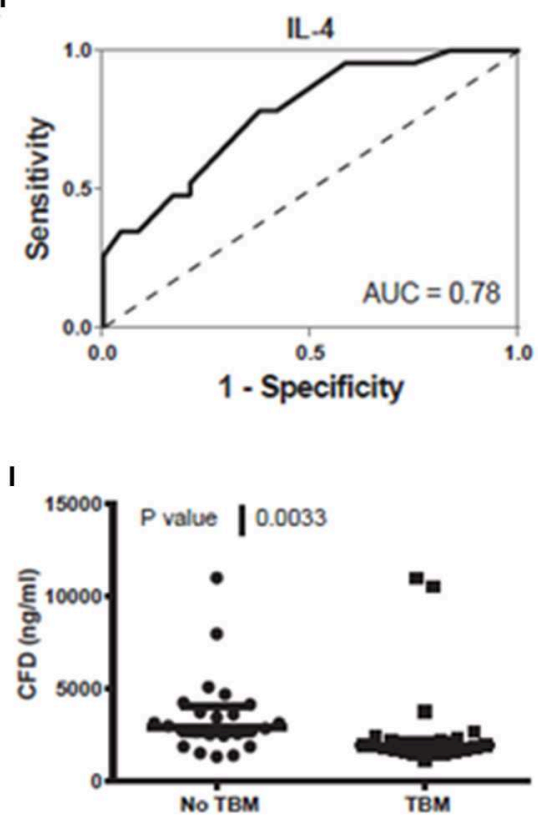

$\mathbf{L}$

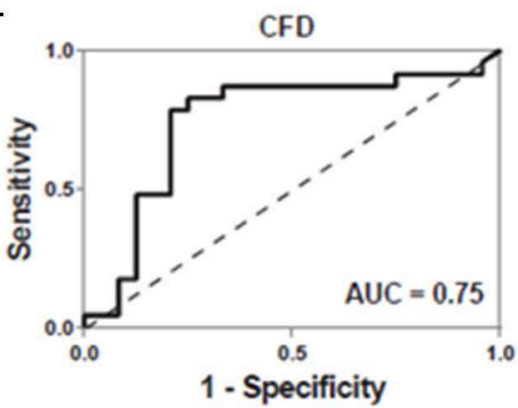

FIGURE 3 | Representative plots showing the concentration of sVCAM-1 (A), CCL2/MCP-1 (B), IL-4 (C), TNF-a (G), CCL4/MIP-1B (H) and CFD (I) detected in serum samples from children with and without TBM and the ROC curves showing the accuracies of sVCAM-1 (D), CCL2/MCP-1 (E), IL-4 (F), TNF-a (J), CCL4/MIP-1B (K) and CFD (L) individually in the diagnosis of TBM. Error bars in the scatter-dot plots indicate the median and inter-quartile ranges. Representative plots for six analytes with $A \cup C \geq 0.75$ are shown. The accuracies of all host biomarkers evaluated in the study are shown in Supplementary Table 1.

IL-13, IL-21, IL-6, MCP-1, IP-10, MIG, GM-CSF, IL-1 $\beta$, IL12/23p40, IL-17A, IL-4, and TNF- $\alpha$ were significantly higher in CSF samples in comparison to serum samples, while the levels of other markers including D-dimer, CC2, CC4b, CC5a, adipsin, CF1, ADAMTS13, cathepsin D, ICAM-1, NCAM1, sVCAM-1, PAI-1, Apo-CIII, CC3, CRP, A1AT, PEDF, SAP, and MIP-4 were significantly higher in serum samples of all study participants compared to CSF (Supplementary Table 2).
Only three proteins (CCL4, G-CSF, and I-309) showed no difference between CSF and serum samples from all children.

\section{DISCUSSION}

We assessed the usefulness of biomarkers comprising a previously identified adult pulmonary TB 7-marker serum 
A

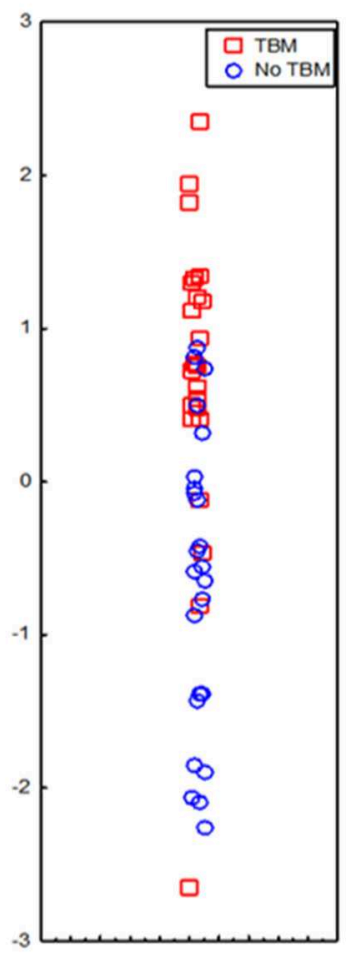

B



FIGURE 4 | Accuracy of the 3-marker serum biosignature (Complement factor D/adipsin, Ab42 and IL-10) in the diagnosis of TBM. Scatter plot showing the ability of the 3-marker signature to classify children as TBM or no TBM (A). ROC curve showing the accuracy of the 3-marker biosignature (B). Red squares: children with TBM; blue circles: children with No TBM.

protein biosignature (28) and modified versions where transthyretin was replaced by NCAM1 and other host biomarkers that have shown potential in the diagnosis of TB disease in recent adult studies, as tools for diagnosis of TBM in children. Although the modified adult 7-marker serum protein biosignature showed potential as a diagnostic tool for TBM in children, we identified a novel smaller TBM-specific 3-marker serum signature (adipsin, $A \beta 42$, and IL-10), which diagnosed childhood TBM with promising accuracy. It is well-known that most of the poor outcomes resulting from being diagnosed with TB are related to difficulties in the diagnosis of the disease and/or delayed initiation of treatment, especially in high burden settings. The currently available diagnostic tests have several shortcomings, especially in young children and in people presenting with extrapulmonary $\mathrm{TB}$, including TBM $(13,14)$. The diagnosis of TBM is particularly challenging in resource-constrained areas owing to the expensive and invasive modalities required in making a proper diagnosis. These tests perform poorly individually $(32,33)$, with a combination of several modalities including clinical presentation, CSF findings, neuroimaging, evidence of extraneural $\mathrm{TB}$, and where possible, mycobacteriological confirmation, required for diagnosis (34). Access to these tests requires admission to a tertiary hospital in relatively well-resourced environments. Consequently, children who do not have access these facilities, especially those in resource-constrained, mainly rural settings, miss the opportunity for early diagnosis. Children living in relatively better-resourced areas such as in the Western Cape Province of South Africa still present at the clinic an average of six times prior to eventual proper diagnosis of TBM (20). These factors emphasize the urgent need for new diagnostic tools in childhood TBM.

Host inflammatory protein biomarkers have been shown to possess potential in the diagnosis of TB disease in both adults and children in previous studies $(25,27,28)$, including being translated into field-friendly point-of-care tests $(22,23)$. In a previous study done in our research group, a 3-marker CSF biosignature (VEGF, IL-13, and LL-37) was identified which showed potential as a tool for the diagnosis of TBM in children (25). In a follow-up study, we validated this biosignature but importantly, showed that replacement of two of the proteins in this signature (IL-13 and LL-37) with new proteins (IFN- $\gamma$ and MPO) resulted in improved accuracy (29). Despite the potential shown by these CSF-based biosignatures, collection of CSF, an invasive procedure that requires lumbar puncture, is challenging in resource-constrained areas. Given the promise shown by blood-based inflammatory biosignatures in pulmonary $\mathrm{TB}$, it is important that similar approaches be assessed for the diagnosis of TBM in children, given that blood will be easier to collect and 
the fact that blood-based tests may be easily converted into finger prick-based tests as its currently being done in another project (www.screen-tb.eu). In the current preliminary study, we have shown proof of principle that blood-based protein biosignatures may be useful in the diagnosis of childhood TBM. Given the limited performance of the adult seven-marker signature in the present study, tests that are developed on adult pulmonary TB patients (e.g., the ScreenTB study) may not perform optimally in children, especially those with TBM as demonstrated in this study. We have however, identified a novel childhood TBM specific 3-marker biosignature which may be considered for further development into a blood-based point-of-care or bedside test for childhood TBM after further validation studies.

A test that is based on blood-based biomarkers such as the biosignature identified in the present study needs to be optimized during future validation and development stages as a screening test for TBM in children. Such a blood-based test is a highpriority need, as identified in the published WHO target product profiles for new diagnostics (35). Further development of such a test into a finger prick blood-based assay will enable easier implementation at the point-of-care or bedside, including in resource-poor settings and may lead to significant reductions in costs, unnecessary lumbar puncture procedures, and the delays that are currently incurred in the diagnosis of TBM in children (20) and consequently, a reduction in the morbidity and mortality currently resulting from TBM. The more invasive and expensive modalities currently being used in the diagnosis of the disease could then only be applied in triaged children with positive point-of-care tests. However, this will only be possible after further validation experiments and incorporation of the biomarkers into such point-of-care test platforms. It is wellknown that host inflammatory biomarker-based tests may not be specific for $\mathrm{TB}$, owing to the expression of the biomarkers in other inflammatory conditions and cancer $(28,36)$. However, it is believed that these specificity concerns may be addressed through the combination of different host biomarkers as done in the present study, with the resultant tests being implemented as triage tests. Given the importance and urgency to commence anti-tuberculous treatment in TBM, a diagnostic test requires adequate sensitivity as well as specificity. A biomarker-based diagnostic test for TBM should have as high sensitivity as possible and preferably above $95 \%$ as recommended by the WHO to be suitable as a triage test (35). Both the modified adult sevenmarker serum TB biosignature and our novel smaller TBMspecific 3-marker serum signature had reasonable sensitivity and specificity when compared to current individual tests for the diagnosis of TBM, with the smaller serum protein signature performing better. However, the accuracies of the two signatures were below the limits specified for a triage test in the WHO target product profiles for new tests (35). As this was largely a proofof-concept study, more work is required to ascertain whether these signatures can be further optimized, for example, with new markers, coupled with ascertaining the best clinical situations where possible tools based on such proteins may be useful in the TBM diagnostic landscape.

One of the proteins that formed part of our new TBM-specific 3-marker serum protein biosignature for samples correlate with increased risk of a neurodegenerative disease known as Alzheimer's disease. However, these levels decrease as disease progresses, supporting the concept of higher accumulation of $A \beta 42$ in neuronal deposits (37). In our study, levels of serum $A \beta 42$ did not show statistical difference between children with TBM and those without TBM. However, these protein contributed to the performance of our TBM-specific 3-marker serum protein biosignature. The association of $\mathrm{A} \beta 42$ and dementia in neurodegenerative disease, its decreased levels in bacterial meningitis (38), and its contribution to our signature might suggest its involvement in the pathology of TBM. IL10 (which also formed part of signature) is a well-known antiinflammatory and regulatory cytokine. The production of IL10 is highly increased in healthy human neonates, making this population to be susceptible to TBM (39). Similarly, serum levels of IL-10 were higher in children without TBM in our study. Adipsin/complement factor D play a role as a regulator in the activation of the alternative complement pathway (40).

The main limitation of the current study was the small sample size, especially small number of individuals with confirmed TBM $(n=3)$. However, as this preliminary study included only children with signs and symptoms and who were referred after strong suspicion of TBM, the design of the study was relatively strong. Although the number of participants enrolled into the study is consistent with patient numbers that were described in multiple previous studies, there is a need to further assess the biomarkers that showed potential in this preliminary study in larger numbers of study participants with suspected meningitis. Such future studies should include children who are HIV infected, and more individuals with confirmed TBM as well as confirmed alternative meningitis. This would allow proper validation of the promising signatures by using training and test sets of samples. The authors of the current manuscript acknowledge the importance of correction of data for multiple comparisons during statistical analysis. This was not done in the in the current study because it was a small, proofof-concept study intended to ascertain whether adult-derived serum biomarkers have potential to be useful in children. More rigorous statistical analysis is recommended in future, wellpowered studies. As HIV-infected individuals and smaller infants may have diminished immune responses as compared to HIVnegative older children and adults (41), it may be worthy to evaluate the effect of HIV status as well as age group (smaller infants vs. older children) on the performance of the biomarkers in future, larger studies. The use of different biosignatures as tools for triaging children enrolled at lower levels of the healthcare system into those with potentially serious conditions such as meningitis, and other less concerning conditions still needs to be investigated and was not the aim of the present preliminary study.

In conclusion, we have shown that a modified version of a previously identified adult 7-marker serum protein biosignature (CRP, SAA, complement factor H, IFN- $\gamma$, IP-10, Apo AI, and NCAM1, in place of transthyretin) may be useful in the diagnosis of TBM in children. However, the performance of this modified signature was reduced, compared to what was reported in adults with pulmonary $\mathrm{TB}$, albeit, with transthyretin in place of NCAM1 in the adult study. Furthermore, we identified a smaller 
childhood TBM specific 3-marker biosignature (adipsin, A $\beta 42$, and IL-10) with potential as a diagnostic tool for childhood TBM. As this was a largely proof-of-concept study, intended to ascertain the utility of serum host protein biomarkers in the diagnosis of childhood TBM, our findings are preliminary and require validation in larger studies.

\section{DATA AVAILABILITY}

The raw data supporting the conclusions of this manuscript will be made available by the authors, without undue reservation, to any qualified researcher.

\section{ETHICS STATEMENT}

This study was carried out in accordance with the recommendations of the Declaration of Helsinki and Good Clinical Practice guidelines, with written informed consent from the parents or legal guardians of all study participants. The parents or legal guardians of all study participants gave written informed consent in accordance with the Declaration of Helsinki. Assent was obtained from children older than 7 years if they had a normal level of consciousness i.e., a Glasgow Coma Score (GCS) of $15 / 15$. The protocol was approved by the Health Research Ethics Committee of the University of Stellenbosch, Tygerberg Academic Hospital (Ethics reference \#: N16/11/142), and the Western Cape Provincial Government.

\section{AUTHOR CONTRIBUTIONS}

NC and GW conceptualized and designed the study and put together the study team, analyzed and interpreted data, and critically revised the manuscript. RS recruited all study participants, analyzed and interpreted data, and critically

\section{REFERENCES}

1. World Health Organization. 10 Facts on Tuberculosis. World Health Organization (2018). Available online at: http://www.who.int/features/ factfiles/tuberculosis/en/ (accessed June 22, 2018).

2. Global Tuberculosis Report. Geneva: World Health Organization (2018). Available online at: http://apps.who.int/medicinedocs/documents/s23553en/ s23553en.pdf (accessed June 7, 2019).

3. Roadmap for Childhood Tuberculosis. Geneva: World Health Organization (2013). Available online at: http://apps.who.int/iris/ bitstream/handle/10665/89506/9789241506137_eng.pdf;jsessionid\$= \$2002C82574A684C603D1F28628353507? sequence $\$=\$ 1$ (accessed May 23, 2018).

4. du Preez K, Schaaf HS, Dunbar R, Walters E, Swartz A, Solomons R, Hesseling AC. Complementary surveillance strategies are needed to better characterise the epidemiology, care pathways and treatment outcomes of tuberculosis in children. BMC Public Health. (2018) 18:397. doi: 10.1186/s12889-018-5252-9

5. Torok ME. Tuberculous meningitis: advances in diagnosis and treatment. $\mathrm{Br}$ Med Bull. (2015) 113:117-31. doi: 10.1093/bmb/ldv003

6. Rock RB, Olin M, Baker CA, Molitor TW, Peterson PK. Central nervous system tuberculosis: pathogenesis and clinical aspects. Clin Microbiol Rev. (2008) 21:243-61. doi: 10.1128/CMR.00042-07

7. Kelekçi S, Karabel M, Karabel D, Hamidi C, Hoşoglu S, Gürkan MF, Taş MA. Bacillus Calmette-Guérin is a preventive factor in mortality revised the manuscript. KS designed and managed the study database, contributed to data analysis, and revised the manuscript. CM processed the samples in the laboratory, contributed to data acquisition, analysis and interpretation, and drafted the manuscript. CS processed the samples in the laboratory, contributed to data acquisition, and revised the manuscript. All authors provided approval for publication of the content, and agreed to be accountable for all aspects of the work.

\section{FUNDING}

This work was supported by the South African Government through the Technology Innovation Agency (TIA), the South African Research Chair Initiative (SARChi) in TB Biomarkers (grant number 86535), the International Collaborations in Infectious Disease Research (ICIDR): Biology and Biosignatures of anti-TB Treatment Response (grant number 5U01IA115619) and the National Research Foundation of South Africa, Grant Number 109437 (RS). The funders were not implicated in the design of the study, sample analysis, interpretation of data, nor the writing of the report and the decision to submit the paper for publication.

\section{ACKNOWLEDGMENTS}

We are grateful to all our study participants and support staff for their contribution to this study.

\section{SUPPLEMENTARY MATERIAL}

The Supplementary Material for this article can be found online at: https://www.frontiersin.org/articles/10.3389/fped. 2019.00376/full\#supplementary-material

of childhood tuberculous meningitis. Int J Infect Dis. (2014) 21:1-4. doi: 10.1016/j.ijid.2013.10.001

8. van Well GTJ, Paes BF, Terwee CB, Springer P, Roord JJ, Donald PR, et al. Twenty years of pediatric tuberculous meningitis: a retrospective cohort study in the Western Cape of South Africa. Pediatrics. (2009) 123:e1-8. doi: 10.1542/peds.2008-1353

9. Ho J, Marais BJ, Gilbert GL, Ralph AP. Diagnosing tuberculous meningitis - have we made any progress? Trop Med Int Health. (2013) 18:783-93. doi: $10.1111 /$ tmi.12099

10. Chiang SS, Khan FA, Milstein MB, Tolman AW, Benedetti A, Starke JR, et al. Treatment outcomes of childhood tuberculous meningitis: a systematic review and meta-analysis. Lancet Infect Dis. (2014) 14:947-57. doi: 10.1016/S1473-3099(14)70852-7

11. Lolachi S, Jp J, Adler D. Tuberculous meningitis, what's new? J Neuroinfect Dis. (2017) 8:248. doi: 10.4172/2314-7326.1000248

12. Perez-Velez CM, Marais BJ. Tuberculosis in Children. N Engl J Med. (2012) 367:348-61. doi: 10.1056/NEJMra1008049

13. Amicosante M, D’Ambrosio L, Munoz M, de Mello FCQ, Tebruegge M, Chegou NN, et al. Current use and acceptability of novel diagnostic tests for active tuberculosis: a worldwide survey. J Bras Pneumol Publicacao. (2017) 43:380-92. doi: 10.1590/S1806-37562017000000219

14. Chegou NN, Hoek KG, Kriel M, Warren RM, Victor TC, Walzl G. Tuberculosis assays: past, present and future. Expert Rev Anti Infect Ther. (2011) 9:457-69. doi: 10.1586/eri.11.23 
15. Bahr NC, Marais S, Caws M, van Crevel R, Wilkinson RJ, Tyagi JS, et al. GeneXpert MTB/Rif to diagnose tuberculous meningitis: perhaps the first test but not the last. Clin Infect Dis. (2016) 62:1133-5. doi: 10.1093/cid/ciw083

16. Bahr NC, Nuwagira E, Evans EE, Cresswell FV, Bystrom PV, Byamukama A, et al. Diagnostic accuracy of Xpert MTB/RIF Ultra for tuberculous meningitis in HIV-infected adults: a prospective cohort study. Lancet Infect Dis. (2018) 18:68-75. doi: 10.1016/S1473-3099(17)30474-7

17. Bahr NC, Tugume L, Rajasingham R, Kiggundu R, Williams DA, Morawski $B$, et al. Improved diagnostic sensitivity for tuberculous meningitis with Xpert(®) MTB/RIF of centrifuged CSF. Int J Tuberc Lung Dis. (2015) 19:1209-15. doi: 10.5588/ijtld.15.0253

18. Berhanu RH, David A, da Silva P, Shearer K, Sanne I, Stevens W, Scott L. Performance of Xpert MTB/RIF, Xpert Ultra, and Abbott RealTi $m$ e MTB for diagnosis of pulmonary tuberculosis in a high-HIV-burden setting. J Clin Microbiol. (2018) 56:e00560-18. doi: 10.1128/JCM.00560-18

19. Arend SM, van Soolingen D. Performance of Xpert MTB/RIF Ultra: a matter of dead or alive. Lancet Infect Dis. (2018) 18:8-10. doi: 10.1016/S1473-3099(17)30695-3

20. Solomons R, Grantham M, Marais BJ, van Toorn R. IMCI indicators of childhood TBM at primary health care level in the Western Cape Province of South Africa. Int J Tuberc Lung Dis. (2016) 20:1309-13. doi: 10.5588/ijtld.16.0062

21. Chegou NN, Walzl G, Bolliger CT, Diacon AH, van den Heuvel MM. Evaluation of Adapted whole-blood interferon-\&gamma; release assays for the diagnosis of pleural tuberculosis. Respiration. (2008) 76:131-8. doi: $10.1159 / 000128575$

22. Corstjens PLAM, Tjon Kon Fat EM, de Dood CJ, van der Ploeg-van Schip JJ, Franken KLMC, Chegou NN, et al. Multi-center evaluation of a user-friendly lateral flow assay to determine IP-10 and CCL4 levels in blood of TB and non-TB cases in Africa. Clin Biochem. (2016) 49:22-31. doi: 10.1016/j.clinbiochem.2015.08.013

23. Sutherland JS, Mendy J, Gindeh A, Walzl G, Togun T, Owolabi O, et al. Use of lateral flow assays to determine IP-10 and CCL4 levels in pleural effusions and whole blood for TB diagnosis. Tuberc Edinb Scotl. (2016) 96:31-6. doi: 10.1016/j.tube.2015.10.011

24. Polivka J, Polivka J, Krakorova K, Peterka M, Topolcan O. Current status of biomarker research in neurology. EPMA J. (2016) 7:14. doi: 10.1186/s13167-016-0063-5

25. Visser DH, Solomons RS, Ronacher K, van Well GT, Heymans MW, Walzl G, et al. Host immune response to tuberculous meningitis. Clin Infect Dis. (2015) 60:177-87. doi: 10.1093/cid/ciu781

26. Rohlwink UK, Mauff K, Wilkinson KA, Enslin N, Wegoye E, Wilkinson RJ, Figaji AA. Biomarkers of cerebral injury and inflammation in pediatric tuberculous meningitis. Clin Infect Dis. (2017) 65:1298-307. doi: $10.1093 / \mathrm{cid} / \mathrm{cix} 540$

27. Jacobs R, Malherbe S, Loxton AG, Stanley K, van der Spuy G, Walzl G, et al. Identification of novel host biomarkers in plasma as candidates for the immunodiagnosis of tuberculosis disease and monitoring of tuberculosis treatment response. Oncotarget. (2016) 7:57581-92. doi: 10.18632 /oncotarget.11420

28. Chegou NN, Sutherland JS, Malherbe S, Crampin AC, Corstjens PLAM, Geluk A, et al. Diagnostic performance of a seven-marker serum protein biosignature for the diagnosis of active TB disease in African primary healthcare clinic attendees with signs and symptoms suggestive of TB. Thorax. (2016) 71:785-94. doi: 10.1136/thoraxjnl-2015-207999

29. Manyelo CM, Solomons RS, Snyders CI, Manngo PM, Mutavhatsindi H, Kriel $\mathrm{B}$, et al. Application of cerebrospinal fluid host protein biosignatures in the diagnosis of tuberculous meningitis in children from a high burden setting. Mediators Inflamm. (2019) 2019:7582948. doi: 10.1155/2019/7582948
30. Marais S, Thwaites G, Schoeman JF, Török ME, Misra UK, Prasad K, et al. Tuberculous meningitis: a uniform case definition for use in clinical research. Lancet Infect Dis. (2010) 10:803-12. doi: 10.1016/S1473-3099(10)70 138-9

31. Fluss R, Faraggi D, Reiser B. Estimation of the Youden Index and its associated cutoff point. Biom J Biom Z. (2005) 47:458-72. doi: 10.1002/bimj.200 410135

32. Thwaites G. Neurological aspects of tropical disease: tuberculous meningitis. J Neurol Neurosurg Psychiatry. (2000) 68:289-99. doi: 10.1136/jnnp.68.3.289

33. Chaidir L, Ganiem AR, vander Zanden A, Muhsinin S, Kusumaningrum T, Kusumadewi I, et al. Comparison of Real Time IS6110-PCR, microscopy, and culture for diagnosis of tuberculous meningitis in a cohort of adult patients in Indonesia. PLoS ONE. (2012) 7:e52001. doi: 10.1371/journal.pone.0052001

34. Marais S, Wilkinson RJ. The diagnosis and medical management of tuberculous meningitis in adults. South Afr Med J. (2014) 104:895. doi: 10.7196/SAMJ.9060

35. World Health Organization. High-Priority Target Product Profiles for New Tuberculosis Diagnostics: Report of a Consensus Meeting. Geneva: World Health Organization (2014). Available online at: https://apps.who.int/ iris/bitstream/handle/10665/135617/WHO_HTM_TB_2014.18_eng.pdf? sequence $=1$ (accessed June 7, 2019).

36. AE-TBC consortium, Chegou NN, Sutherland JS, Namuganga A-R, Corstjens PL, Geluk A, et al. Africa-wide evaluation of host biomarkers in QuantiFERON supernatants for the diagnosis of pulmonary tuberculosis. Sci Rep. (2018) 8:2675. doi: 10.1038/s41598-018-20855-7

37. Findeis MA. The role of amyloid $\beta$ peptide 42 in Alzheimer's disease. Pharmacol Ther. (2007) 116:266-86. doi: 10.1016/j.pharmthera.2007.06.006

38. Sjögren M, Gisslén M, Vanmechelen E, Blennow K. Low cerebrospinal fluid $\beta$-amyloid 42 in patients with acute bacterial meningitis and normalization after treatment. Neurosci Lett. (2001) 314:33-6. doi: 10.1016/S0304-3940(01)02285-6

39. Isabel BE, Rogelio HP. Pathogenesis and Immune response in tuberculous meningitis. Malays J Med Sci. (2014) 21:4-10.

40. Stanton CM, Yates JRW, den Hollander AI, Seddon JM, Swaroop A, Stambolian D, et al. Complement factor D in age-related macular degeneration. Invest Ophthalmol Vis Sci. (2011) 52:8828-34. doi: 10.1167/iovs.11-7933

41. Vanden Driessche K, Persson A, Marais BJ, Fink PJ, Urdahl KB. Immune Vulnerability of Infants to Tuberculosis. Clin Dev Immunol. (2013) 2013:1-16. doi: $10.1155 / 2013 / 781320$

Conflict of Interest Statement: NC, CM, GW, and RS are listed as inventors on a South African Provisional Patent Application No. 2018/03410, entitled "Cerebrospinal fluid (CSF) and blood based biomarkers for diagnosing tuberculous meningitis". NC and GW are listed as inventors on another patent application (PCT/IB2015/052751) entitled "Method for diagnosing tuberculous meningitis." These applications are pending.

The remaining authors declare that the research was conducted in the absence of any commercial or financial relationships that could be construed as a potential conflict of interest.

Copyright (c) 2019 Manyelo, Solomons, Snyders, Mutavhatsindi, Manngo, Stanley, Walzl and Chegou. This is an open-access article distributed under the terms of the Creative Commons Attribution License (CC BY). The use, distribution or reproduction in other forums is permitted, provided the original author(s) and the copyright owner(s) are credited and that the original publication in this journal is cited, in accordance with accepted academic practice. No use, distribution or reproduction is permitted which does not comply with these terms. 